\title{
Anabases
}

ANABASES Traditions et réceptions de l'Antiquité

$14 \mid 2011$

Varia

\section{Jacqueline FABRE-SERRIS, Rome, l'Arcadie et la mer des Argonautes. Essai sur la naissance d'une mythologie des origines en Occident}

Cristina Noacco

\section{OpenEdition}

Journals

Édition électronique

URL : http://journals.openedition.org/anabases/2396

DOI : 10.4000/anabases.2396

ISSN : 2256-9421

Éditeur

E.R.A.S.M.E.

Édition imprimée

Date de publication : 1 octobre 2011

Pagination : 260-263

ISSN : 1774-4296

\section{Référence électronique}

Cristina Noacco, « Jacqueline fabre-SerRIs, Rome, l'Arcadie et la mer des Argonautes. Essai sur la

naissance d'une mythologie des origines en Occident », Anabases [En ligne], 14 | 2011, mis en ligne le 01 octobre 2011, consulté le 22 septembre 2020. URL : http://journals.openedition.org/anabases/2396 DOI : https://doi.org/10.4000/anabases.2396

Ce document a été généré automatiquement le 22 septembre 2020

(c) Anabases 


\title{
Jacqueline FABRE-SERRIS, Rome, l'Arcadie et la mer des Argonautes. Essai sur la naissance d'une mythologie des origines en Occident
}

\author{
Cristina Noacco
}

\section{RÉFÉRENCE}

Jacqueline FABRE-SERRIS, Rome, l'Arcadie et la mer des Argonautes. Essai sur la naissance d'une mythologie des origines en Occident, Villeneuve d'Ascq, Presses Universitaires du Septentrion, 2008, $250 \mathrm{p}$.

23 euros / ISBN : 978-2-7574-0034-0.

1 L'ouvrage de J. Fabre-Serris représente une enquête sur l'élaboration d'un double mythe romain des origines au $\mathrm{I}^{\mathrm{er}}$ siècle av. J.-C., lors de la crise sociale et économique que Rome a connue à l'issue des guerres puniques. Ce mythe des origines se fonde sur deux images: celle de l'Arcadie, la première terre habitée, et celle de la mer des Argonautes, la première mer traversée. Tout au long de son étude, J. Fabre-Serris prouve que si le substrat mythologique et géographique de ces images est profondément grec, son application et son fonctionnement relèvent incontestablement de la culture romaine.

2 Le livre se compose de deux volets, qui correspondent aux deux éléments étudiés du mythe romain des origines, d'un cahier iconographique, d'une bibliographie et de deux index fort utiles (le premier recense les auteurs et les œuvres anciennes cités, tandis que le deuxième, général, comprend également les noms mythologiques et les concepts évoqués).

Dans la première partie du volume, intitulée «Les Arcadies romaines» (p. 11-162), J. Fabre-Serris fait état des variantes que le mythe de l'Arcadie originelle a connues à 
travers les textes latins. Elle retrace avant tout l'histoire de la construction d'un mythe des origines grecques de l'Vrbs, à travers les témoignages d'auteurs tels que Denys d'Halicarnasse, Virgile et Ovide, qui reconnaissent l'Arcadie comme le lieu d'origine des premiers colons du Latium. Pour Denys d'Halicarnasse et pour Virgile, Évandre était le chef d'une ancienne colonie d'Arcadiens, implantée sur les collines du Latium. C'est lui qui, dans l'Énéide, raconte à Énée «ce que Rome fut avant Rome » (p. 16) : une nation pieuse et guerrière, valeurs que les Romains revendiquent comme spécifiques à leur culture. Dans les Fastes, Ovide explique différemment l'arrivée des Arcadiens dans le Latium, mettant en relation Faunus, Hercule et une famille romaine, les Fabii, en raison des rapports privilégiés de cette dernière avec le dieu Faunus, qui est identifié à Pan. J. Fabre-Serris passe ensuite en revue la poésie lyrique, qu'elle considère comme un prélude à la peinture mythifiée de l'Arcadie pastorale. Apparue dans les Hymnes homériques, cette dernière est développée dans le De rerum Natura de Lucrèce et dans l'œuvre poétique d'Ovide, mettant en scène le dieu Pan, l'inventeur de la musique et, par conséquent, de la poésie pastorale. Tibulle et Properce, poètes élégiaques, récupèrent la leçon de Lucrèce, en y ajoutant deux éléments qui en étaient exclus : la «fable » et l'amour. J. Fabre-Serris étudie ensuite l'origine du mythe arcadien de Rome dans les idylles et les épigrammes pastorales grecques, qui opposaient aux préoccupations guerrières de l'épopée le plaisir du chant et de l'amour. Gallus et Virgile (notamment dans Bucolique 2 et 10) sont les poètes qui mieux que tout autre ont témoigné de cette appropriation d'un genre poétique grec pour favoriser l'idéalisation des origines de Rome, à travers un récit fictionnel fondé sur la nostalgie et sur la triade « mort, compassion et souvenir » (p. 105) propre au mythe arcadien.

Dans un autre volet de son enquête sur les variantes romaines du mythe de l'Arcadie, J. Fabre-Serris insiste sur le lien étroit qui unit l'homme et la nature à la fois dans la littérature et dans la peinture du $\mathrm{I}^{\mathrm{er}}$ siècle av. J.-C. Elle rapproche ainsi la figure du bouvier mythique grec Daphnis, célébré après sa mort (dans la Bucolique 5 de Virgile) comme l'inventeur du genre poétique, de la peinture de la Maison de Jason, où Pan est accompagné de trois figures féminines près d'un tombeau. Dans la Bucolique 5 , comme dans le livre 2 des Géorgiques, le paysan a remplacé le berger en tant que détenteur du savoir sur la nature, comme condition d'une vie heureuse, tandis que l'amour et les passions en général sont exclus des occupations pastorales, qui ruinent la concorde. Curae et otia fondent la vie du paysan, comme ils fondaient, chez Lucrèce, la vie des premiers hommes. Ces deux ouvrages mettent en scène, à la fin du I $\mathrm{I}^{\mathrm{er}}$ siècle av. J.-C., le topos de l'éloge de la vie rustique. Telle est la version, typiquement romaine, d'après $\mathrm{J}$. Fabre-Serris, du mythe de l'âge d'or arcadien. Le bonheur appartient à l'homme de la campagne, qui refuse les mirages des passions et cultive l'idéal du "cœur pur " (p. 109). L'ancrage géographique et le nom de l'Arcadie ont dès lors été vidés de leur réalité, pour donner vie à un mythe «identifiable seulement par ses images» (p. 112). Cela explique l'engouement de la peinture augustéenne pour les paysages arcadiens, appelés également "sacro-idylliques » et l'importance du jardin à la même époque, témoignant du désir de renouer un contact perdu avec la nature, une nature qui entretient avec l'art une relation étroite, la première offrant le modèle à la deuxième qui, à son tour, tend à établir les règles de la beauté naturelle. Les enjeux de l'art des jardins sont en particulier évidents dans l'œuvre de Longus (Daphnis et Chloé) : le jardin offre des fleurs et des fruits, un abri aux oiseaux, et un lieu de pensée, selon la leçon de Platon, aux hommes. Dans un dernier chapitre de la première partie de son étude, l'auteur évoque les récits mythologiques qu'Ovide et d'autres poètes latins situent en 
Arcadie. Il s'agit des histoires de Daphné, Io, Syrinx, Callisto, Cornix, Aréthuse et Scylla, ainsi que d'une version latine de l'histoire de Polyphème.

5 Les p. 130-136, auxquelles il faut associer les remarques des p. 105-106 et 155-158, offrent une belle description et une subtile interprétation de quelques fresques connues de l'époque augustéenne, reproduites en couleur dans l'ouvrage. La lecture de ces représentations iconographiques du mythe de l'Arcadie complètent et confirment les conclusions auxquelles J. Fabre-Serris parvient dans le domaine littéraire : elle y reconnaît la naissance de la première « notion de paysage » (p. 165) et affirme qu'« à la fin de la République, la nostalgie de la nature sauvage, où les dieux se mêlaient aux hommes, redonne de la force à l'imaginaire romain des origines et le mythe arcadien sert à repenser les grands motifs et les valeurs du mos maiorum » (p. 127).

La deuxième partie du livre, consacrée au mythe de «la mer des Argonautes " (p. 163-214), retrace l'histoire d'un autre exemple, complémentaire du premier, d'appropriation et d'adaptation d'un récit mythique grec aux enjeux idéologiques romains. Il s'agit du récit de la traversée de la Méditerranée par les Argonautes, pour la conquête de la Toison d'or. Ce récit a permis de donner à Rome " une autre image des commencements et de penser l'histoire de son propre destin » (p. 165). J. Fabre-Serris présente tout d'abord les témoignages littéraires de l'expédition des Argonautes, dont le plus ancien est le Carmen 64 de Catulle, et les analyse avec beaucoup de précision, en soulignant notamment, dans le texte indiqué, les images qui permettent de décrire la navigation et le domaine inquiétant de la mer à l'aune de la traversée familière des plaines (p. 167-168; pour l'ex., le terme currus, « char », désigne le navire, tandis que la formule caerula aequora, "les plaines azurées", indique la vaste étendue de la mer). Viennent ensuite les textes d'Ennius, inspiré par Euripide, qui insiste sur la valeur positive du voyage, et de Cicéron, qui évoque un passage des Argonautiques d'Apollonius de Rhodes. Le texte de Catulle, quant à lui, présente la particularité de raconter «les dernières noces de l'âge d'or » (p. 173) : celles de Thétis et Pélée, d'une immortelle avec un mortel. C'est bien lors de ce mariage que la Discorde jeta entre les déesses une pomme, entraînant la rupture de la communion entre les dieux et les hommes. Dès lors, l'expédition des Argonautes marque un tournant décisif dans l'histoire de l'humanité. Horace, dans l'Épode 16, nie même toute possibilité d'un retour de l'âge d'or: la poésie latine ne peut célébrer désormais que des valeurs perdues et, en particulier, la uirtus et la pietas.

7 J. Fabre-Serris se penche également sur la valeur que les auteurs latins ont attribuée à la première navigation : pour Sénèque et Valerius Flaccus, elle est liée à la prouesse des jeunes guerriers grecs, défiant le monde inconnu de la mer. Mais d'autres auteurs, sur les traces d'Ennius, y voient l'origine de la guerre et des commerces funestes, qui entraînent la corruption des valeurs morales. Un témoignage de cette conception se trouve chez Lucrèce, pour qui le navire Argo, construit avec les pins du Pélion, devient le symbole de la perdition et de la fin de l'âge d'or. Virgile (Bucolique 4) et Tibulle (Élégie 1,3) reprennent cette idée et Ovide va jusqu'à réprouver le départ du navire (Mét., 1, 94-96). Ensuite, J. Fabre-Serris étudie la manière dont les auteurs latins ont utilisé le mythe de la traversée des Argonautes pour réfléchir sur la fin de l'âge d'or de l'humanité primitive. Sénèque, dans le deuxième chœur de Médée, considère la première navigation comme une audace excessive. Il affirme même que le voyage des «pins du Pélion » (métonymie désignant le navire Argo) a relié entre elles des terres (le Pélion et la Colchide), qui avaient auparavant toujours été séparées par la mer. Par là, 
Argo a rompu les principes du bonheur originel et de l'ordre du monde, les foedera mundi, faute qui est due, d'après Sénèque, au fait d'« avoir violé l'espace intouché de la mer » (p. 197) et qui a causé, à travers la rencontre de Jason et de Médée, la destruction des liens familiaux, fondateurs de toute société.

Dans un dernier chapitre de son étude, J. Fabre-Serris illustre la manière dont le récit mythologique de la mer des Argonautes a pu devenir un symbole du destin de l'humanité. C'est l'épopée de Valerius Flaccus qui, dans son prooemium, exalte un aspect du mythe qui avait été critiqué par la tradition poétique romaine: la première traversée de la mer. La conquête de la Toison d'or n'est, pour lui, qu'un prétexte. De plus, Jason ne doit pas être condamné pour son audace, mais considéré comme un héros que les dieux ont investi d'une mission. Rome s'y identifie. Mieux, elle superpose son rêve d'expansion en Méditerranée à la mission de conquête de l'inconnu qu'a remplie Jason. Elle se donne pour but la conquête et l'acculturation du monde. J. FabreSerris remarque que le nom de l'empereur ayant produit un tel dessein n'est pas prononcé. Mais elle ajoute qu'il suffit au lecteur averti de Valerius Flaccus de se souvenir du prooemium et de l'adresse de l'auteur à son empereur pour y reconnaître un éloge de la politique expansionniste d'Auguste.

9 L'auteur du livre conclut son étude par l'examen du mythe de l'Arcadie et de la mer des Argonautes dans la poésie $\mathrm{du} \mathrm{xx}^{\mathrm{e}}$ siècle. Elle cite tout d'abord deux poèmes de Lawrence, The Argonautes et Middle of World. Le premier est marqué par la nostalgie du monde des origines, héritée de l'esprit romain, tandis que le deuxième permet la rencontre des deux mythes analysés, celui de la première mer et celui de la première terre. Elle cite aussi Cesare Pavese, qui a lui aussi évoqué la mer des Argonautes dans ses Dialoghi con Leucò : on y retrouve à la fois les motifs de Catulle (Carmen 64) et la rupture des foedera mundi de Sénèque (Médée). Elle cite enfin deux auteurs français: Giono, qui avait identifié sa Provence à l'Arcadie, et Camus, qui décrit les Noces de l'homme avec la nature primordiale, métamorphose ultime de l'idée d'Arcadie au moment de la rupture la plus tragique de l'accord entre l'homme et la nature, au $\mathrm{xx}^{\mathrm{e}}$ siècle. La poésie redécouvre ainsi, dans l'entre-deux-guerres, le lien indissoluble qui unit l'homme à l'animal et au végétal. La réflexion sur l'essence et sur le destin de l'homme n'en sort que renforcée.

10 Le double parcours que propose J. Fabre-Serris à travers la tradition latine des deux mythes des origines étudiés plonge le lecteur au cœur du questionnement qui investit la civilisation romaine en crise sur son identité et sur ses spécificités. Il offre un exemple d'appropriation et d'adaptation de l'héritage mythologique grec à ses buts idéologiques et permet de comprendre le regard que Rome a porté sur son passé et sur son avenir au ${ }^{\text {er }}$ siècle av. J.-C., entre le temps perdu du bonheur primordial et la mission de conquête du monde méditerranéen qu'elle s'attribue.

$11 \mathrm{Si}$, d'un côté, la lecture de cet ouvrage demande au public non latiniste une attention toujours éveillée, afin de suivre l'évolution de la pensée qui le soutient à travers de nombreuses références aux sources antiques, de l'autre, J. Fabre-Serris a su transmettre à ses lecteurs le goût pour la quête des origines qui a fondé son étude. Il serait ainsi tentant de poursuivre l'enquête de l'auteur, soit en direction d'autres éléments mythologiques ayant pu servir à la construction de l'identité romaine de l'époque augustéenne, soit en réfléchissant aux métamorphoses de l'Arcadie et d'Argo en dehors du cadre spatio-temporel analysé. 


\section{AUTEURS}

\section{CRISTINA NOACCO}

Université de Toulouse (UTM)

cnoacco@yahoo.fr 